Check for updates

Cite this: Phys. Chem. Chem. Phys., 2019, 21, 7874

Received 15th February 2019, Accepted 21st March 2019

DOI: 10.1039/c9cp00923j

rsc.li/pccp

\title{
Optical revelation of defects in epitaxial barium titanate films $\dagger$
}

\author{
M. Tyunina, $\mathbb{D} \star^{a b}$ D. Chvostova ${ }^{b}$ and A. Dejneka ${ }^{b}$
}

\begin{abstract}
Allying epitaxial strain and synthesis conditions allows for the introduction of specific point defects in perovskite oxide films. In ferroelectric films, such defects lead to essential polar and electronic properties, which can enable advanced applications. Here, to elucidate the nature of the defects, optical constants are investigated in the spectral range of $0.7-8.8 \mathrm{eV}$ in epitaxial ferroelectric $\mathrm{BaTiO}_{3}$ films, which are synthesized under different conditions. It is demonstrated that oxygen-vacancy-related defects are responsible for a peculiar transition below the bandgap at $\sim 2.7-2.9 \mathrm{eV}$ and significant blueshifts of $\sim 0.3-0.4 \mathrm{eV}$ of the gap and the main interband transitions. These observations suggest that the defects are dipolar complexes comprising titanium cations and oxygen vacancies $\left(\mathrm{Ti}^{3+}-\mathrm{V}_{\mathrm{O}}\right)$.
\end{abstract}

\section{Introduction}

The high potential of epitaxial perovskite oxide films for advanced electronic, photonic, and spintronic applications motivates extensive continuous studies of the synthesis and fundamentals of such films., ${ }^{1,2}$ In perovskite oxides, strong effects and response functions are intrinsically related to distortions of the perovskite cell and, as such, are determined by cationic composition. Importantly, these materials are capable of accepting high concentrations of cationic dopants, which allow for the adjustment of the material's properties. Often, heterovalent doping is associated with the formation of oxygen vacancies, which are widely accepted as the most common defects in perovskite oxides. ${ }^{3}$ In addition to the perovskitespecific cationic control of functional responses, epitaxial films enable the tailoring of their properties using substrate-induced lattice strain and interfacial phenomena. ${ }^{1,2}$ In recent years, it was also recognized that for epitaxial films, oxygen vacancies could be produced by a combined action of strain and synthesis conditions. ${ }^{4-11}$ In particular, using relatively low oxygen pressure during pulsed laser deposition (PLD) of strained ferroelectric $\mathrm{BaTiO}_{3}$ (BTO) resulted in epitaxial films of proper cationic stoichiometry, but exhibiting anomalous unit-cell expansion, low electrical resistivity, and/or enhanced polarization. ${ }^{4-9}$ This BTO behavior was ascribed to oxygen vacancies or other charged defects of ambiguous nature. ${ }^{8,9}$ Here, to get better insight into

\footnotetext{
${ }^{a}$ Microelectronics Research Unit, Faculty of Information Technology and Electrical Engineering, University of Oulu, P. O. Box 4500, FI-90014, Finland. E-mail: marina.tjunina@oulu.fi

${ }^{b}$ Institute of Physics of the Czech Academy of Sciences, Na Slovance 2, 18221 Prague, Czech Republic

$\dagger$ Electronic supplementary information (ESI) available. See DOI: 10.1039/ с9ср00923j
}

the presence, type, and possible origin of defects in epitaxial BTO films grown by PLD at reduced oxygen pressure, we investigate spectra of optical constants of such low-pressure films. As a reference, we study a bulk BTO crystal and highpressure BTO films. The detected impacts of oxygen-vacancyrelated defects include an optical transition at photon energies below the bandgap and a profound global shift of the gap and interband transitions to higher energies. These observations are discussed in terms of in-gap states and a large internal electric field, which are produced by dipolar complexes of titanium cations and oxygen vacancies.

\section{Experimental}

Epitaxial perovskite BTO films with a thickness of $\sim 40 \mathrm{~nm}$ were grown by pulsed laser deposition. Single-crystal epitaxially polished (001) STO substrates were used (MTI Corp.). For electrical characterization, epitaxial bottom electrode layers of $\mathrm{SrRuO}_{3}$ (SRO) and Pt top pads were formed by PLD. The BTO and SRO films were deposited at a temperature of $973 \mathrm{~K}$. The low-pressure and high-pressure BTO films were prepared using oxygen pressure of 0.1-2.0 $\mathrm{Pa}$ and $20 \mathrm{~Pa}$, respectively. The SRO films were grown at $20 \mathrm{~Pa}$. Post-deposition cooling of the highpressure films was performed in oxygen atmosphere. During this procedure the oxygen pressure was gradually increased to $10^{5} \mathrm{~Pa}$. The cooling of the low-pressure films was conducted at the deposition pressure. The crystal structure of the films was inspected by high-resolution X-ray diffraction (XRD) on a Bruker D8 DISCOVER diffractometer using $\mathrm{Cu} \mathrm{K} \alpha$ radiation. The in-plane and out-of-plane lattice parameters of the films were determined from the positions of diffraction peaks using STO as a reference. The ferroelectric properties were 
characterized using a TF 2000E Analyzer (aixACCT Systems $\mathrm{GmbH}$ ). In these measurements, the electric field was applied and the response was acquired along the out-of-plane direction of the SRO/BTO/Pt stacks. The optical properties of the BTO films, the reference polydomain randomly oriented BTO crystal, and the crystal STO substrate were studied using variable angle spectroscopic ellipsometry, which is a precise technique for determining optical properties of materials at the nanoscale. We note that capabilities of such methods as luminescence, electron spin resonance, and deep-level transient spectroscopies are often insufficient for studies of defects in thin ferroelectric films because of substrate contributions, small amount of material, and temperature dependent electrical properties of the films. On the contrary, spectroscopic ellipsometry enables studies of very thin films on eventually arbitrary substrates. The studies were carried out on a J. A. Woollam ellipsometer at photon energies of (0.75-8.8) eV, at room temperature, and in dry nitrogen atmosphere. The ellipsometric spectra were acquired at incident angles of 65 and $70 \mathrm{deg}$, with the energy step of $0.02 \mathrm{eV}$. The dielectric functions and optical constants of the films and crystals (BTO and STO) were extracted from the spectra of ellipsometric angles using a commercial WVASE32 software package. ${ }^{12}$ More details on the used ellipsometric procedures can be found elsewhere. ${ }^{13,14}$

\section{Results and discussion}

The theoretical BTO/STO lattice misfit is $\left[s=a_{\mathrm{S}} / a_{0}-1\right]$, where $a_{\mathrm{S}}$ is the lattice parameter of STO and $a_{0}$ is the lattice parameter of a cubic cell of BTO $\left(a_{0}=V^{1 / 3}\right.$ for unit-cell volume $\left.V\right)$. The estimated biaxial in-plane misfit strain is compressive, with its magnitude larger than $2.5 \%$. Assuming complete relaxation of such a large misfit during BTO growth, one can expect a room-temperature in-plane tensile strain of $\sim 0.3 \%$, which arises due to mismatch in thermal expansion coefficients of BTO and STO. ${ }^{15}$

$\mathrm{XRD}$ analyses showed a cube-on-cube-type epitaxial growth of both the high-pressure and low-pressure BTO films [Fig. 1(a and b)]. The BTO films are perovskite-structure and possess epitaxial relationships $[100](001) \mathrm{BTO} \|[100](001)$ STO. The measured room-temperature lattice parameters evidence biaxial in-plane elongation and out-of-plane shrinkage of unit cell in the high-pressure BTO film, in agreement with the estimated thermal strain. On the contrary, the low-pressure films possess strongly expanded unit cells, which are elongated in the out-ofplane direction. The unit-cell volume of the low-pressure films is $2-3 \%$ larger than in the bulk. To outline films' structure, we use the ratio of the out-of-plane lattice parameter to the in-plane parameter: the ratio is approximately $1.03,1.02$, and 0.99 for pressures of $0.1,2.0$, and $20 \mathrm{~Pa}$, respectively. The crystal structure of our films is consistent with that reported before and suggests the formation of oxygen-vacancy-related point defects in the low-pressure films. ${ }^{5-9}$

The ferroelectric observations also agree with previous studies. $^{7-9,16}$ The measured quasistatic polarization-voltage
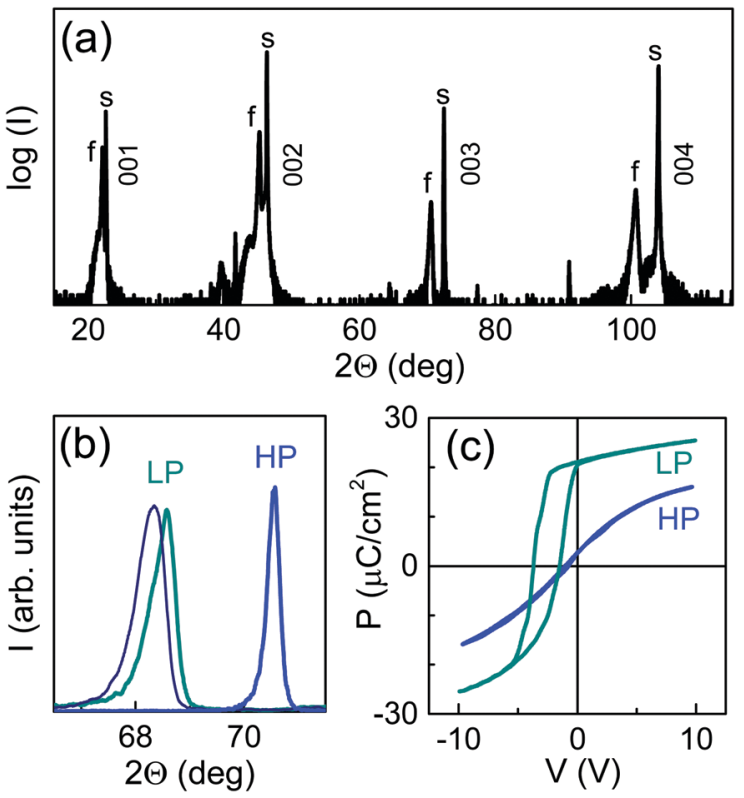

Fig. 1 (a) Typical $\Theta-2 \Theta$ X-ray diffraction pattern in epitaxial BTO films. Diffraction peaks from the film and substrate are marked by " $f$ " and " $s$ ", correspondingly. (b) $\Theta-2 \Theta$ scan around (003) perovskite diffraction in the low-pressure (marked by LP) and high-pressure (marked by HP) films. (c) Quasistatic polarization-voltage loops in the low-pressure (marked by LP) and high-pressure (marked by HP) films.

loops indicate strongly imprinted out-of-plane polarization in the low-pressure films compared to the in-plane polarization and polarization rotation in the high-pressure films [Fig. 1(c)]. The large imprinted polarization confirms the presence of defects in the low-pressure BTO films. ${ }^{8}$

Because oxygen-vacancy-related defects can produce in-gap states in BTO, the type of the defects may be deduced from electronic excitations and optical transitions from/to these states. Therefore, the optical properties of the low-pressure and high-pressure BTO films and of the reference polydomain randomly oriented BTO crystal are analysed next.

As seen from the absorption spectra [Fig. 2(a)], all BTO samples are transparent in the visible spectral range, exhibit abrupt absorption edges at photon energies $E>3 \mathrm{eV}$, and possess broad absorption peaks around $5 \mathrm{eV}$. Closer inspection reveals an unexpected shift of the absorption edge to higher photon energies (blueshift) in the low-pressure films compared to the reference crystal and high-pressure film [Fig. 2(b)]. The Tauc plots evidence indirect gaps in all samples [Fig. 2(c)]. ${ }^{17,18}$ The bandgap energies $E_{\mathrm{g}}$ were found from the linear fits $\left[(\alpha E)^{1 / 2} \propto\left(E-E_{\mathrm{g}}\right)\right]$, where $\alpha$ is the absorption coefficient. Compared to the crystal $\left(E_{\mathrm{g}} \approx 3.20 \mathrm{eV}\right)$, the low-pressure films demonstrate a strong blueshift of $\sim 0.3 \mathrm{eV}$ in the bandgaps. A very weak redshift only is seen in the high-pressure film. Also, surprisingly, the main absorption peak is blueshifted in the low-pressure films. Importantly, a broad pre-edge hump in absorption is seen in the defects-containing low-pressure films.

The blueshifts and the pre-edge feature are better expressed in the spectra of the real and imaginary parts of the dielectric function, $\varepsilon_{1}$ [Fig. 3(a)] and $\varepsilon_{2}$ [Fig. 3(b)], correspondingly. 

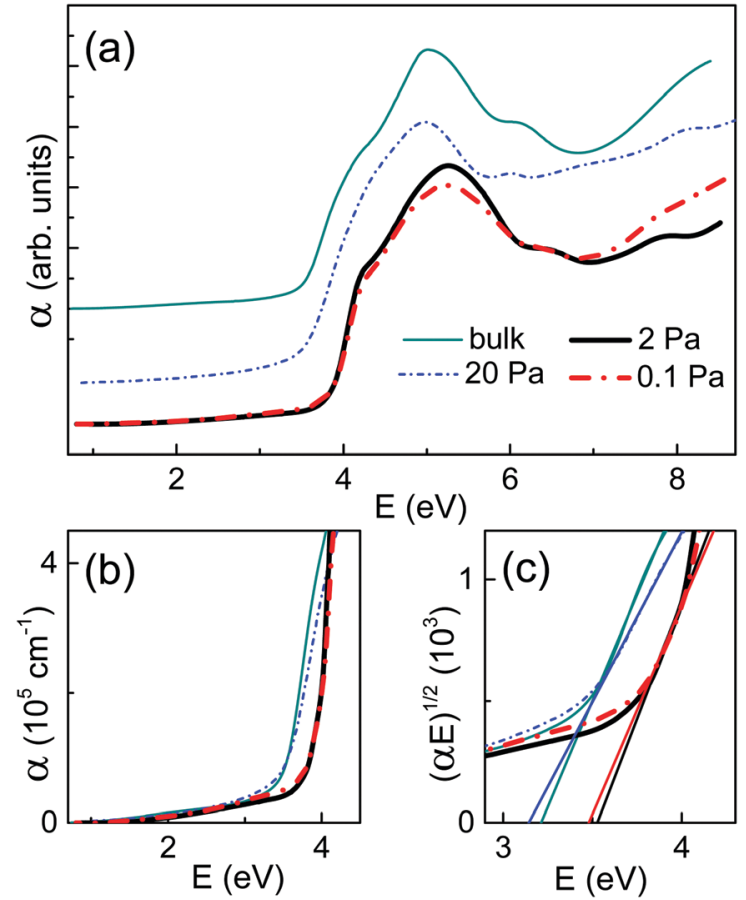

Fig. 2 (a and b) Absorption coefficient $\alpha$ as a function of photon energy and (c) Tauc plots for indirect bandgap in the low-pressure films (0.1 Pa and $2 \mathrm{~Pa}$ ), high-pressure film (20 Pa), and crystal (bulk). Straight lines are fits in (c).
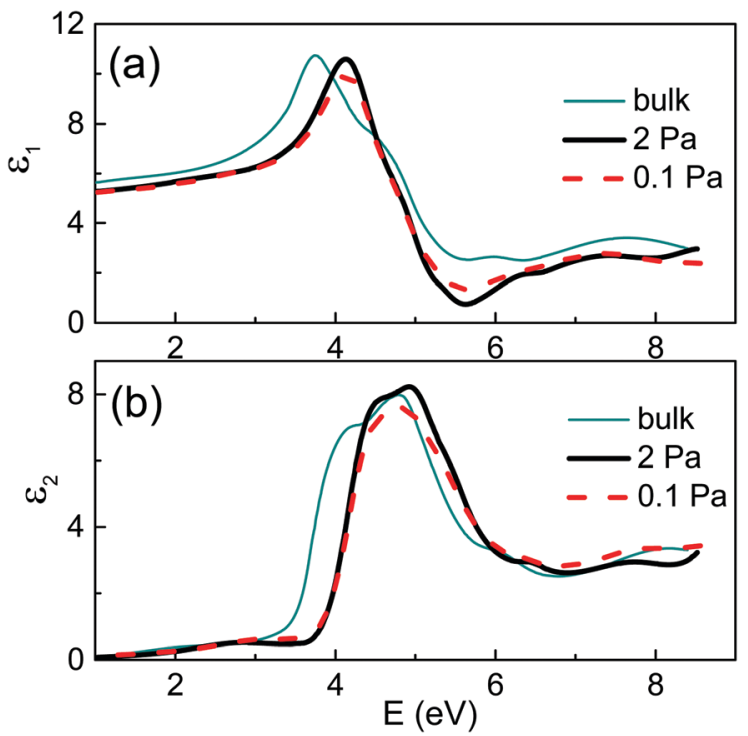

Fig. 3 Spectra of (a) real and (b) imaginary parts of the dielectric function in the low-pressure films and crystal.

The broad peaks in optical spectra [Fig. (2 and 3)] result from several electronic excitations, or transitions (e.g., from the top valence bands to the bottom conduction bands). To explicitly determine the energies of these transitions, we apply a critical point (CP) approach. ${ }^{19,20}$ This approach links singularities in the electronic density of states with spectral lines in the dielectric function. The energies $E_{0}$ and the phase angles $\phi$ of the spectral lines, or CPs, were obtained from the second derivatives of the dielectric functions as described before. ${ }^{14}$ For simplicity and ease of comparison, the phase angles were assumed $\phi=0,0.5,1$, or $1.5 \pi$ in expression (1) for a twodimensional CP:

$$
\frac{\mathrm{d}^{2} \varepsilon}{\mathrm{d} E^{2}}=\frac{A \exp (\mathrm{i} \phi)}{\left(E-E_{0}+\mathrm{i} \Gamma\right)^{2}} .
$$

Here $A$ and $\Gamma$ are the amplitude and width of the line, correspondingly.

The full spectra of derivatives are displayed in ESI. $\dagger$ The main CPs are illustrated in Fig. 4.

Compared to the reference bulk crystal and high-pressure film, the low-pressure films demonstrate an additional preedge $\mathrm{CP}$ around 2.7-2.9 eV [Fig. 4(a and b)] and significant blueshifts of the main CPs [Fig. 4(c-j)]. In particular, a strong $\mathrm{CP}$ at $\sim 3.7-3.8 \mathrm{eV}$ in the reference samples is shifted to $\sim 4.2 \mathrm{eV}$ in the low-pressure films [Fig. 4(c and d)]. Moreover, the higher-energy fine structure (another CP at $\sim 4.2 \mathrm{eV}$ ) near to this reference CP [Fig. 4(d)] is smeared out in the lowpressure films [Fig. 4(c)]. Concurrently, a broad reference CP at $\sim 5.3-5.4 \mathrm{eV}$ is transformed into a fine-structured one at higher energies in the low-pressure films [Fig. 4(g and h)]. Also a CP at $\sim 6.1 \mathrm{eV}$ in the crystal is moved to $6.3-6.4 \mathrm{eV}$ in the low-pressure films [Fig. 4(i and j)]. However, a strong CP at $\sim 4.9 \mathrm{eV}$ does not experience noticeable transformations in the low-pressure films, unlike the other CPs. We note that the reference CPs agree well with the previous experimental studies of bulk BTO. ${ }^{21-23}$

The CP analysis shows defect-induced global changes in the band structure of BTO. First, the pre-edge CP attests the presence of deep in-gap states. Previously, an onset of absorption in the pre-edge spectral region of $\sim 2.0-2.6 \mathrm{eV}$ was attributed to oxygen vacancies in the hydrogen-reduced BTO crystals. ${ }^{24} \mathrm{How}^{-}$ ever, recent studies of hydrogenated BTO showed that the defects are not simply oxygen vacancies but neutral centers, each containing two hydrogen ions at a doubly charged oxygen vacancy. ${ }^{25}$ Therefore, the CP at $\sim 2.7-2.9 \mathrm{eV}$ cannot be directly related to oxygen vacancies in the low-pressure films.

Concurrently, the energies of the gap and of the main optical transitions increase by $\sim 0.3-0.4 \mathrm{eV}$ in the low-pressure films. These films experience enormous expansion of crystal unit cell, or large tensile strain. The lattice expansion should have led to the narrowing of the bandgap and redshifts of the interband transitions, ${ }^{26,27}$ which oppose the experimental observations. To resolve this controversy, we note the voltage offset in the polarization-voltage loop [Fig. 1(c)]. The offset signifies an in-built potential up to $3 \mathrm{~V}$. Thus, an internal electric field is up to $10^{7}-10^{8} \mathrm{~V} \mathrm{~m}^{-1}$ in the low-pressure films. Because the electric field is known to raise and split the lowest conduction bands in perovskite oxide ferroelectrics, ${ }^{28,29}$ the internal electric field can produce the observed blueshifts.

As a cause of the internal electric field in the low-pressure PLD-prepared epitaxial BTO films, an out-of-plane alignment of charged defects was hypothesized. ${ }^{8}$ In BTO, oxygen vacancy can be nominally doubly charged or can bind one electron 


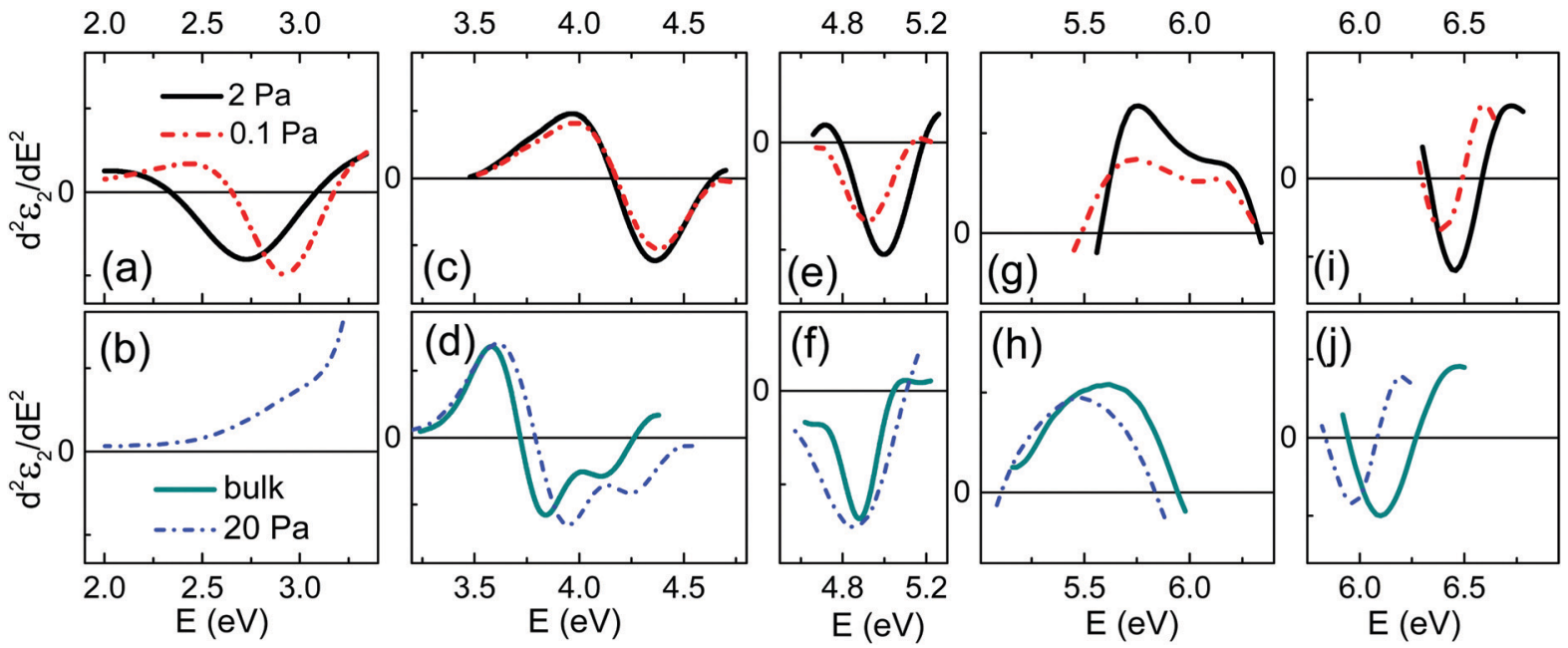

Fig. 4 Second derivative $d^{2} \varepsilon_{2} / d E^{2}$ as a function of photon energy in the low-pressure films (upper panels a, c, e, g, i) and reference high-pressure film and crystal (lower panels $b, d, f, h, j$ ).

(charged vacancy) or two electrons (neutral vacancy). Because lattice expansion favors stability of neutral vacancies, ${ }^{30,31}$ stabilization of charged vacancies is unlikely in the lowpressure films. We note that the formation of a charged oxygen vacancy $\mathrm{V}_{\mathrm{O}}$ can be associated with electron transfer to a Ti $\mathrm{e}_{\mathrm{g}}$ orbital, leading to a dipolar complex $\left(\mathrm{Ti}^{3+}-\mathrm{V}_{\mathrm{O}}\right) \cdot{ }^{32-34}$ Interestingly, the pre-edge $\mathrm{CP}$ is consistent with the reported excitation of such complexes by irradiation with $E>2.5 \mathrm{eV}^{32}$ Furthermore, the $\left(\mathrm{Ti}^{3+}-\mathrm{V}_{\mathrm{O}}\right)$ complex is characterized by an anisotropic anomalous expansion of the $\mathrm{TiO}_{6}$ octahedron and, correspondingly, symmetry lowering. ${ }^{34}$ Here, the lattice expansion of the low-pressure films corroborates the octahedral distortion related to the $\left(\mathrm{Ti}^{3+}-\mathrm{V}_{\mathrm{O}}\right)$ complexes. The outof-plane elongation of the low-pressure films matches the out-of-plane orientation of octahedral elongations, which are

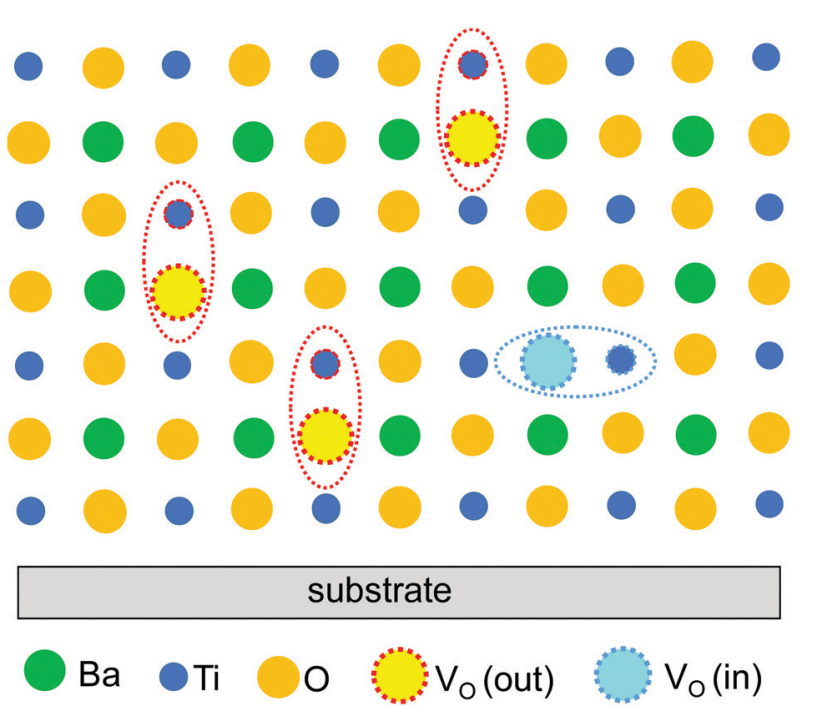

Fig. 5 Schematics of the out-of-plane and in-plane dipolar $\left(\mathrm{Ti}^{3+}-\mathrm{V}_{\mathrm{O}}\right)$ complexes in epitaxial cube-on-cube-type film. likely produced by the out-of-plane alignment of the $\left(\mathrm{Ti}^{3+}-\mathrm{V}_{\mathrm{O}}\right)$ defects [Fig. 5].

Thus, both the pre-edge $\mathrm{CP}$ and the blueshifts of the gap and main CPs are coherent with the presence and out-of-plane alignment of the dipolar $\left(\mathrm{Ti}^{3+}-\mathrm{V}_{\mathrm{O}}\right)$ defects in the low-pressure films. More investigations are still required to clarify the exact nature, lattice distortions, electronic structure, stability, and formation of these defects. In particular, such methods as positron annihilation spectroscopy and electron spin resonance can be especially valuable for these investigations.

Besides the presence and optical impact of defects, our studies revealed that along with the blueshifts of the bandgaps and main CPs, the strong $\mathrm{CP}$ at $\sim 4.9 \mathrm{eV}$ is not affected by the electric field. BTO is known to possess the lower and higher subbands at the bottom of conduction band, or LCB and HCB for brevity. Considering a field-induced lift-up of LCB, the transitions from the valence band (VB) to LCB should blueshift, while those to HCB should not change. ${ }^{28,29}$ The changes of the main CPs $(\sim 3.7,4.2$, and $5.4 \mathrm{eV})$ confirm their VB-LCB assignment, and the stability of the $\mathrm{CP}$ at $\sim 4.9 \mathrm{eV}$ verifies its VB-HCB assignment. ${ }^{21}$ However, the blueshift of the $\mathrm{CP}$ at $\sim 6.1 \mathrm{eV}$ points to a VB-LCB transition rather than a traditionally assumed VB-HCB one.

\section{Conclusions}

In conclusion, oxygen-vacancy-related defects are studied by investigation of optical constants in a broad spectral range of 0.75-8.8 ev in epitaxial ferroelectric $\mathrm{BaTiO}_{3}$ films. It is demonstrated that the defects produce in-gap states and internal electric field, which are manifested by an additional optical transition below the gap and significant blueshifts of the interband transitions, correspondingly. The formation and out-of-plane alignment of dipolar $\left(\mathrm{Ti}^{3+}-\mathrm{V}_{\mathrm{O}}\right)$ defect complexes can be responsible for this behavior. 


\section{Conflicts of interest}

There are no conflicts to declare.

\section{Acknowledgements}

The authors are thankful to T. Kocourek, M. Jelinek, and O. Pacherova for sample preparation and structural characterization. The authors acknowledge support from the Czech Science Foundation (Grant No. 19-09671S) and the European Structural and Investment Funds and the Ministry of Education, Youth and Sports of the Czech Republic through Programme "Research, Development and Education”' (Project No. SOLID21 - CZ.02.1.01/ 0.0/0.0/16_019/0000760).

\section{References}

1 Epitaxial Growth of Complex Metal Oxides, ed. G. Koster, M. Huijben and G. Rijnders, Woodhead Publishing, Cambridge, 2015.

2 Multifunctional Oxide Heterostructures, ed. E. Y. Tsymbal, E. R. A. Dagotto, C.-B. Eom and R. Ramesh, Oxford University Press, Oxford, 2012.

3 C. Ang, Z. Yu and L. E. Cross, Phys. Rev. B: Condens. Matter Mater. Phys., 2000, 62, 228.

4 H. Shigetani, K. Kobayashi, M. Fujimoto, W. Sugimura, Y. Matsui and J. Tanaka, J. Appl. Phys., 1997, 81, 693.

5 T. Zhao, F. Chen, H. Lu, G. Yang and Z. Chen, J. Appl. Phys., 2000, 87, 7442.

6 J. Shin, S. V. Kalinin, A. Y. Borisevich, E. W. Plummer and A. P. Baddorf, Appl. Phys. Lett., 2007, 91, 202901.

7 A. P. Chen, F. Khatkhatay, W. Zhang, C. Jacob, L. Jiao and H. Wang, J. Appl. Phys., 2013, 114, 124101.

8 A. R. Damodaran, E. Breckenfeld, Z. Chen, S. Lee and L. W. Martin, Adv. Mater., 2014, 26, 6341.

9 M. Li, J. Zhou, X. Jing, M. Zeng, S. Wu, J. Gao, Z. Zhang, X. Gao, X. Lu, J. M. Liu and M. Alexe, Adv. Electron. Mater., 2015, 1500069.

10 U. Aschauer and N. A. Spaldin, Appl. Phys. Lett., 2016, 109, 031901.

11 A. Herklotz, D. Lee, E. J. Guo, T. L. Meyer, J. R. Petrie and H. N. Lee, J. Phys.: Condens. Matter, 2017, 29, 493001.

12 Guide to Using WVASE 32: Spectroscopic Ellipsometry Data Acquisition and Analysis Software, J. A. Woollam, Inc., 2008.

13 A. Dejneka, D. Chvostova, O. Pacherova, T. Kocourek, M. Jelinek and M. Tyunina, Appl. Phys. Lett., 2018, 112, 031111.
14 M. Tyunina, L. D. Yao, D. Chvostova, T. Kocourek, M. Jelinek, A. Dejneka and S. van Dijken, New J. Phys, 2015, 17, 043048.

15 V. B. Shirokov, Yu. I. Yuzyuk, B. Dkhil and V. V. Lemanov, Phys. Rev. B: Condens. Matter Mater. Phys., 2007, 75, 224116.

16 M. Tyunina and M. Savinov, Phys. Rev. B, 2016, 94, 054109.

17 J. Tauc, R. Grigorovici and A. Vancu, Phys. Status Solidi, 1966, 15, 627.

18 S. Piskunov, E. Heifets, R. I. Eglitis and G. Borstel, Comput. Mater. Sci., 2004, 29, 165.

19 P. Lautenschlager, M. Garriga, S. Logothetidis and M. Cardona, Phys. Rev. B: Condens. Matter Mater. Phys., 1987, 35, 9174.

20 P. Lautenschlager, M. Garriga, L. Vina and M. Cardona, Phys. Rev. B: Condens. Matter Mater. Phys., 1987, 36, 4821.

21 D. Bäuerle, W. Braun, V. Saile, G. Sprtissel and E. E. Koch, Z. Phys. B: Condens. Matter Quanta, 1978, 29, 179.

22 M. Cardona, Phys. Rev., 1965, 140, A651.

23 S. G. Choi, A. M. Dattelbaum, S. T. Picraux, S. K. Srivastava and C. J. Palmstrøm, J. Vac. Sci. Technol., B: Microelectron. Nanometer Struct.--Process., Meas., Phenom., 2008, 26, 1718.

24 J. Y. Chang, C. R. Chinjen, R. H. Tsou, C. Y. Huang, C. C. Sun and M. W. Chang, Opt. Commun., 1997, 138, 101.

25 T. U. Ito, A. Koda, K. Shimomura, W. Higemoto, T. Matsuzaki, Y. Kobayashi and H. Kageyama, Phys. Rev. B, 2017, 95, 020301(R).

26 R. F. Berger, C. J. Fennie and J. B. Neaton, Phys. Rev. Lett., 2011, 107, 146804.

27 F. Wang, I. Grinberg and A. M. Rappe, Appl. Phys. Lett., 2014, 104, 152903.

28 J. R. Brews, Phys. Rev. Lett., 1967, 18, 662.

29 M. DiDomenico, Jr. and S. H. Wemple, J. Appl. Phys., 1969, 40, 720 .

30 M. Choi, F. Oba and I. Tanaka, Appl. Phys. Lett., 2011, 98, 172901.

31 D. S. Aidhy, B. Liu, Y. Zhang and W. J. Weber, Comput. Mater. Sci., 2015, 99, 298.

32 R. Scharfschwerdt, A. Mazur, O. F. Schirmer, H. Hesse and S. Mendricks, Phys. Rev. B: Condens. Matter Mater. Phys., 1996, 54, 15284.

33 V. V. Laguta, A. M. Slipenyuk, I. P. Bykov, M. D. Glinchuk, M. Maglione, D. Michau, J. Rosa and L. Jastrabik, Appl. Phys. Lett., 2005, 87, 022903.

34 E. Erdem, P. Jakes, R. A. Eichel, D. C. Sinclair, M. Pasha and I. M. Reaney, Funct. Mater. Lett., 2010, 3, 65. 